\title{
On Lyman-line asymmetries in quiescent prominences ${ }^{\star}$
}

\author{
S. Gunár ${ }^{1,2}$, P. Heinzel ${ }^{1,2}$, U. Anzer ${ }^{2}$, and B. Schmieder ${ }^{3}$ \\ 1 Astronomical Institute, Academy of Sciences of the Czech Republic, 25165 Ondřejov, Czech Republic \\ e-mail: [gunar; pheinzel]@asu.cas.cz \\ 2 Max-Planck-Institut für Astrophysik, Karl-Schwarzschild-Strasse 1, 85740 Garching, Germany \\ 3 Observatoire de Paris, Section de Meudon, 92195 Meudon Principal Cedex, France
}

Received 5 May 2008 / Accepted 8 July 2008

\section{ABSTRACT}

\begin{abstract}
Aims. We study the asymmetries of the synthetic hydrogen Lyman lines and the process responsible for their formation. Methods. To obtain the synthetic Lyman line profiles, we use a multi-thread prominence fine-structure model consisting of identical 2D threads. The 2D thread models are in MHS equilibrium, include an empirical prominence-corona transition region, and solve consistently 2D non-LTE radiative transfer. Each thread of the multi-thread model has a randomly assigned line-of-sight (LOS) velocity.

Results. The synthetic Lyman spectrum obtained by multi-thread modelling exhibits substantial asymmetries of the line profiles, even though the LOS velocities of individual threads are only of the order of $10 \mathrm{~km} \mathrm{~s}^{-1}$. Moreover, our results indicate that the synthetic Lyman- $\alpha$ profiles may exhibit an opposite asymmetry to that of the higher Lyman lines.

Conclusions. The presence and behaviour of the asymmetrical profiles of the synthetic Lyman lines agree with observed profiles acquired by SUMER.
\end{abstract}

Key words. Sun: prominences - radiative transfer - line: profiles

\section{Introduction}

Hydrogen Lyman lines and continuum observations provide a considerable amount of information about the structure and physical properties of quiescent prominences. They represent an important constraint for the prominence fine-structure modelling and a useful tool for spectroscopic diagnostics of the prominence fine structures. The SUMER UV-spectrograph (Solar Ultraviolet Measurements of Emitted Radiation - Wilhelm et al. 1995) onboard SOHO (Solar and Heliospheric Observatory) has provided a substantial amount of quiescent-prominence spectral data in Lyman lines. Some of these data and their analyses can be found in Gunár et al. (2006, 2007b); Heinzel et al. (2001, 2006); Schmieder et al. (1999, 2007), and in a review of SOHO prominence observations by Patsourakos \& Vial (2002; see also a review of the prominence fine structures by Heinzel 2007).

Proper interpretation of observed Lyman lines requires sophisticated models of the prominence plasma in a magnetic field that include the PCTR (Prominence-Corona Transition Region), and complex non-LTE radiative transfer computations. Heasley \& Mihalas (1976) constructed 1D prominence models in magnetohydrostatic (MHS) equilibrium of the Kippenhahn-Schlüter type (Kippenhahn \& Schlüter 1957) with multi-level non-LTE radiative transfer computations. Similar 1D models were constructed by Gouttebroze et al. (1993). The importance of including the PCTR in prominence modelling was demonstrated by Anzer \& Heinzel (1999) and, for He I triplet lines, by Labrosse $\&$ Gouttebroze (2004). Two-dimensional models of the prominence fine structures were presented by Heinzel \& Anzer (2001). These 2D thread models in MHS equilibrium included an empirical PCTR and solved consistently 2D multi-level non-LTE

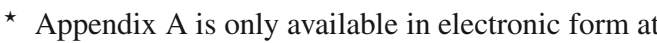
http://www . aanda. org
}

radiative transfer (see also Heinzel \& Anzer 2005). The analyses of the synthetic Lyman lines obtained by 2D modelling of the prominence fine structures were presented by Heinzel et al. (2005) and the Lyman continuum analysis by Gunár et al. (2007a).

Studies of the synthetic Lyman spectrum have focused mainly on symmetrical Lyman line profiles. To our knowledge, no non-LTE model has considered asymmetrical profiles in quiescent prominences. However, substantial asymmetries of the Lyman lines were observed already during the OSO-8 (Orbiting Solar Observatory) mission (Vial 1982). Observations acquired by the SUMER spectrograph exhibit a considerable number of asymmetrical profiles of Lyman lines, as reported by Heinzel et al. (2001) \& Schmieder et al. (1999, 2007). These significant asymmetries of the lower members of the Lyman line series cannot be explained by pure Doppler shifts because the line-of-sight (LOS) velocities in quiescent prominences are too small, of the order of $10 \mathrm{~km} \mathrm{~s}^{-1}$ (Tandberg-Hanssen 1995; Kucera et al. 1999; Madjarska et al. 1999; Wiik et al. 1999). A statistical study of the Lyman- $\alpha$ and Lyman- $\beta$ lines observed by SUMER (Vial et al. 2007) demonstrated that asymmetrical profiles occur in several places within the observed quiescent prominence. These authors also showed that the asymmetries of the Lyman lines can have opposite senses at the same position in the prominence (e.g. a dominant blue peak of one Lyman line coincides with a dominant red peak in a different spectral line at the same position in the prominence).

In a previous paper (Gunár et al. 2007b), we employed a multi-thread modelling method that adopted a set of 2D prominence fine-structure thread models. We achieved a robust fit of the synthetic Lyman spectrum with selected observed symmetrical profiles. However, we found that the observed Lyman spectrum typically consists of a number of asymmetrical profiles of 
the Lyman lines as also found by Vial et al. (2007). In the present paper, we use our multi-thread modelling method to study the origin of asymmetries of the Lyman line profiles.

The paper is organized as follows. Section 2 presents the multi-thread model of the prominence fine structures, both static and models with the LOS velocities. Section 3 shows the resulting synthetic spectra and the formation of the line-profile asymmetries and Sect. 4 presents the discussion and our conclusions.

\section{Multi-thread prominence fine-structure models}

Our multi-thread models consist of a set of identical 2D prominence fine structures which were described by Heinzel \& Anzer (2001). These 2D fine-structure threads are vertically infinite and the variation of all physical quantities occurs in a horizontal plane parallel to the solar surface. The 2D MHS-equilibrium of these fine-structure models was discussed by Heinzel \& Anzer (2001), where these authors assumed an empirical temperature profile. The temperature structure is characterized by two different shapes of the PCTR. Within a narrow PCTR layer across the magnetic field lines the temperature exhibits a steep gradient between the central coolest part of the prominence and its boundaries. In contrast, the temperature increases gradually along the field lines within a rather extended PCTR layer. This difference could be due to the large difference of the heat conduction along and across the magnetic field. For a detailed description of the 2D prominence fine-structure thread models, we refer the reader to Heinzel \& Anzer (2001) and Heinzel et al. (2005).

The multi-thread prominence fine-structure models that we use consist of $N$ identical 2D threads without any mutual radiative interaction. Individual threads are arranged perpendicularly to the LOS along individual field lines (Fig. 1). Each thread is randomly shifted with respect to the foremost thread. The LOS therefore intersects individual threads at different positions along the length of each 2D thread, which produces different emerging line profiles, optical thicknesses, etc., in contrast to multi-thread $1 \mathrm{D}$ slab models (e.g. Fontenla et al. 1996). This method was developed by Gunár et al. (2007b), who showed that the synthetic spectra generated by multi-thread modelling provide a closer description of observed Lyman line profiles than spectra produced by single-thread 2D models. We note that the models used by Gunár et al. (2007b) were static and that the synthetic spectra produced were therefore symmetrical.

\subsection{Multi-thread models with random LOS velocities}

The modelling of asymmetrical Lyman line profiles, often found in prominence data, requires the presence of the LOS velocities of the prominence fine structures. The LOS velocities can be produced by flows of prominence plasma along the magnetic field lines or by oscillations of the magnetic structure. In this paper, we consider the LOS velocities to be ad-hoc quantities. However, in the case of flows, the LOS must have some inclination with respect to the magnetic field lines to provide a significant Doppler component.

We modified our, previously static, multi-thread finestructure models by randomly assigning a LOS velocity $\left(\xi^{(n)}\right)$ to each thread. We therefore first computed the radiative transfer in a single $2 \mathrm{D}$ thread model, and the effect of the LOS velocities on the spectral line profiles was introduced by the Doppler shifts of the profiles emerging from individual threads and the Doppler-shifted absorption of the radiation from the background threads. A schematic diagram of the multi-thread model with

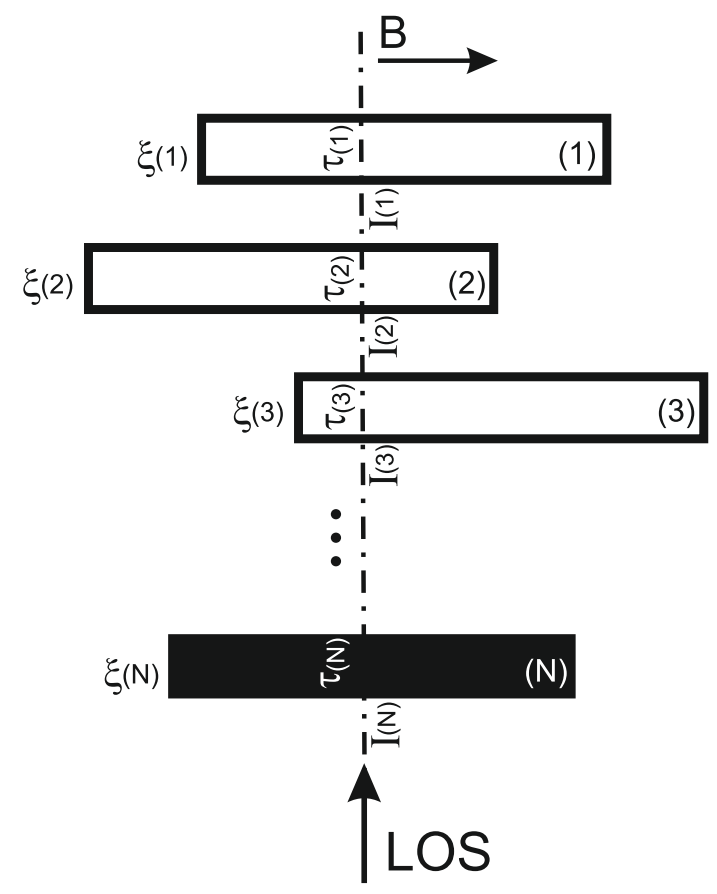

Fig. 1. Scheme of the multi-thread model with $N$ randomly shifted 2D threads and randomly assigned LOS velocities $\left(\xi^{(n)}\right)$. B is the horizontal field component.

randomly assigned velocities for individual threads is shown in Fig. 1. The total emerging intensity from such a multi-thread model at a given wavelength and position along the length of the foremost thread (thread $N$ ) in the observers frame is described by the formula

$$
\begin{aligned}
I_{\text {total }}(\lambda)= & {\left[\ldots\left[\left[I_{\lambda^{(1)}} \exp \left(-\tau_{\lambda^{(2)}}\right)+I_{\lambda^{(2)}}\right] \times \exp \left(-\tau_{\lambda^{(3)}}\right)+I_{\lambda^{(3)}}\right]\right.} \\
& \left.\times \ldots+I_{\lambda^{(N-1)}}\right] \times \exp \left(-\tau_{\lambda^{(N)}}\right)+I_{\lambda^{(N)}},
\end{aligned}
$$

where $I_{\lambda^{(n)}}$ and $\tau_{\lambda^{(n)}}$ are defined as follows

$$
I_{\lambda^{(n)}} \equiv I\left(\lambda-\Delta \lambda^{(n)}\right)
$$$$
\tau_{\lambda^{(n)}} \equiv \tau\left(\lambda-\Delta \lambda^{(n)}\right) \text {. }
$$

Here $\Delta \lambda^{(n)}$ is the Doppler shift for the thread $n$ corresponding to its LOS velocity $\xi^{(n)}$ given by

$\Delta \lambda^{(n)}=\lambda_{0} \frac{\xi^{(n)}}{c}$

where $\lambda_{0}$ represents the line-centre wavelength of a given spectral line and $\xi^{(n)}$ is the LOS velocity of thread $n$. Positive values of $\xi^{(n)}$ represent velocities towards the observer. Equation (1) is identical to Eq. (5) from Gunár et al. (2007b) but here we use a different mathematical formulation that follows closely the numerical scheme of the present multi-thread model. In contrast to Gunár et al. (2007b), we use also a reversed numbering of threads. The intensity $I_{\lambda^{(n)}}$ emerges from a single thread. This is obtained by solving a non-LTE radiative transfer problem within a 2D fine-structure thread illuminated by the solar surface (see Heinzel \& Anzer 2001). We note that, for $I$ and $\tau$, we do not provide subscripts indicating the position along the thread explicitly (as in Gunár et al. 2007b) but we do consider random shifts of individual threads.

\section{Synthetic spectra}

In the present theoretical investigation, we chose a set of 10 identical threads randomly shifted with respect to the foremost thread 
and with randomly assigned LOS velocities from an interval of $\langle-10,10\rangle \mathrm{km} \mathrm{s}^{-1}$. Velocities of the order of $10 \mathrm{~km} \mathrm{~s}^{-1}$ do not significantly affect the source function, therefore the radiative transfer computations for the individual 2D thread do not consider the velocity fields. We also neglect the effect of flows on partiallycoherent scattering (PRD), which we consider for the Lyman- $\alpha$ and Lyman- $\beta$ lines as in previous papers. We have used the same parameters of the 2D thread model as in Gunár et al. (2007b), i.e. the central (minimum) temperature $T_{0}=7000 \mathrm{~K}$, the boundary (maximum) temperature $T_{\mathrm{tr}}=10^{5} \mathrm{~K}$, the maximum column mass in the centre of the thread $M_{0}=1.1 \times 10^{-4} \mathrm{~g} \mathrm{~cm}^{-2}$, the horizontal field strength in the middle of the thread $B_{x}(0)=6$ Gauss, and the boundary pressure $p_{0}=0.015 \mathrm{dyn} \mathrm{cm}^{-2}$. The width of the thread $(1000 \mathrm{~km})$ is chosen arbitrarily, and the length of the thread (approximately $28000 \mathrm{~km}$ ) is determined by the MHS equilibrium computations.

To illustrate quantitatively the behaviour of the derived synthetic spectral lines, we generated a single realization of the multi-thread model. The actual set of LOS velocities of this realization was $\xi=\{7,-7,9,-2,-7,-9,4,-3,4,-7\} \mathrm{km} \mathrm{s}^{-1}$ and the actual set of shifts of positions along the length of the thread, with respect to the foremost thread (thread 10), was $\{2,2,12,4,-6,-4,31,12,-27,0\}$ for threads 1 to 10 , where the positions were measured in units of $340 \mathrm{~km}$. Figure 2 shows the arrangement of the actual realization of the multi-thread model. Individual threads are drawn according to their positional shifts with respect to the foremost thread with arrows indicating the direction and magnitude of LOS velocities. White solid-line contours represent the iso-contours of the density $(5.0,2.5,1.0,0.5$, $0.25,0.1$, and $0.0510^{-13} \mathrm{~g} \mathrm{~cm}^{-3}$, starting from the centre of the thread) and the grey-scale represents the scale of the temperature from the minimum central temperature (black) to the maximum boundary temperature (white). The dot-dashed line represents the centre of the foremost thread (position 42) and the thin solid lines indicate the positions 20 to 60 .

The combined effect of Doppler-shifted line profiles and optical-depth profiles together with the effect of positional shifts between individual threads produce synthetic spectra with significant asymmetries of the peak intensities. Figure 3 shows the resulting line profiles emerging from the foremost thread (thread 10 in our notation) of this specific multi-thread model. The profiles of Lyman- $\alpha$ to Lyman- $\gamma$ emerging at the positions 23, 34, 38, and 40 along this thread are shown. The Lyman- $\delta$ line exhibits similar behaviour to the Lyman- $\beta$ and Lyman- $\gamma$ lines and it is therefore not included in Fig. 3. Each column represents one spectral line. The full lines represent the profiles generated by the 10 thread model with random LOS velocities. For reference, dot-dashed lines show the profiles that would be produced by a single-thread model. All profiles for positions 23 to 42 are displayed in the on-line content in Figs. 6 to 9 with position 42 denoting the mid-point of the foremost thread. In contrast to the single-thread model represented by dotdashed line profiles, the resulting multi-thread model profiles exhibit significant asymmetries. The asymmetries of the multithread synthetic profiles of each Lyman line vary with position along the thread rather randomly. Moreover, the predominance of the red or blue peak of the synthetic profiles changes between different spectral lines at a given position along the thread. This can be seen, for example, at position 23 where the blue peak is dominant for Lyman- $\alpha$ and the red peak for the other lines. A similar behaviour is visible at the positions 34 and 38 where the Lyman- $\alpha$ profile is symmetrical, while the higher lines are rather asymmetric. The predominance of one peak above another within asymmetrical line profiles tends to be more pronounced for higher members of the Lyman series.

We note that the present realization of the multi-thread model is purely arbitrary. However, we studied also a number of other random realizations, for which the asymmetrical profiles showed a similar behaviour.

\subsection{The nature of asymmetrical profiles}

The significant asymmetries of the synthetic Lyman line profiles, shown in Figs. 3 and A.1 to A.4, cannot be attributed entirely to Doppler shifts of the line profiles. If we consider, for example, the Lyman- $\beta$ line, then a LOS velocity of the order of $10 \mathrm{~km} \mathrm{~s}^{-1}$ would provide a Doppler shift of approximately $0.03 \AA$. The line width itself however is around $0.4 \AA$, and the shift of the line profile would therefore be lower than $10 \%$ of its width.

Instead of being an entirely Doppler-shift effect, the asymmetrical profiles are produced by the combined effect of various Doppler shifts of the emission line profiles and the absorption coefficient profiles (optical thickness profiles) of the individual threads. We also found that the emission peaks were rather sharp and narrow and the absorption profiles considerably steep around the positions of peaks. This leads in many cases to strongly asymmetrical absorption. Moreover, the differences in opacities of the Lyman- $\alpha$, Lyman- $\beta$, and higher Lyman lines can even produce opposite asymmetries in these lines. Figure 4 shows the details of this formation process for the Lyman- $\beta$ line profile at position 40 . The presentation follows the numerical formula given by Eq. (1). The first plot in Fig. 4 shows the line profile emerging from the last thread (thread 1, solid line). The intensity is scaled in all plots to $10^{-10} \mathrm{erg} \mathrm{s}^{-1} \mathrm{~cm}^{-2} \mathrm{sr}^{-1} \mathrm{~Hz}^{-1}$. The dashed line represents the optical depth of the following thread (thread 2) drawn to the same scale. The arrow sign represents the absorption of the line profile emerging from the previous thread (thread $n$ ) by the following thread (thread $n+1)$, given by $\left[I_{\lambda^{(n)}} \times \exp \left(-\tau_{\left.\lambda^{(n+1)}\right)}\right)\right.$, where $I_{\lambda^{(n)}}$ and $\tau_{\lambda^{(n)}}$ are defined by Eqs. (2) and (3). The second plot in the first row shows the result of the absorption and the third plot in the first row shows the line profile emerging from the thread 2 . The sum of these two line profiles is shown by the solid line in the first plot in the second row. The process of profile generation thus propagates through the entire multi-thread set. The first plot in the last row on the right hand side shows the resulting line profile emerging from the foremost thread (thread 10). The second plot in the last row on the right hand side represents the instrumental profile of the SUMER A detector. The third plot shows the final line profile after convolution with the instrumental profile. The instrumental profile is Gaussian with a standard deviation $\sigma$ equivalent to one SUMER A detector spectral pixel, which corresponds to $0.043 \AA$. This agrees with the results of Chae et al. (1998), who measured the instrumental FWHM (full width at half maximum) of the SUMER A detector to be approximately 2.3 detector pixels. The FWHM for the Gaussian distribution is equal to approximately $2.4 \sigma$. We note that the optical depth profiles in Fig. 4 (dashed lines) are drawn to the same scale as the intensity profiles. Only sections of the optical depth profiles with $\tau_{\lambda} \leq 5$ are therefore drawn. The full optical depth profiles for Lyman- $\alpha$ to Lyman- $\delta$ at selected positions along the length of the thread are shown in Fig. A.5. 


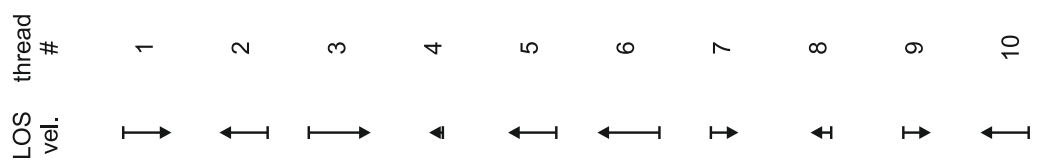

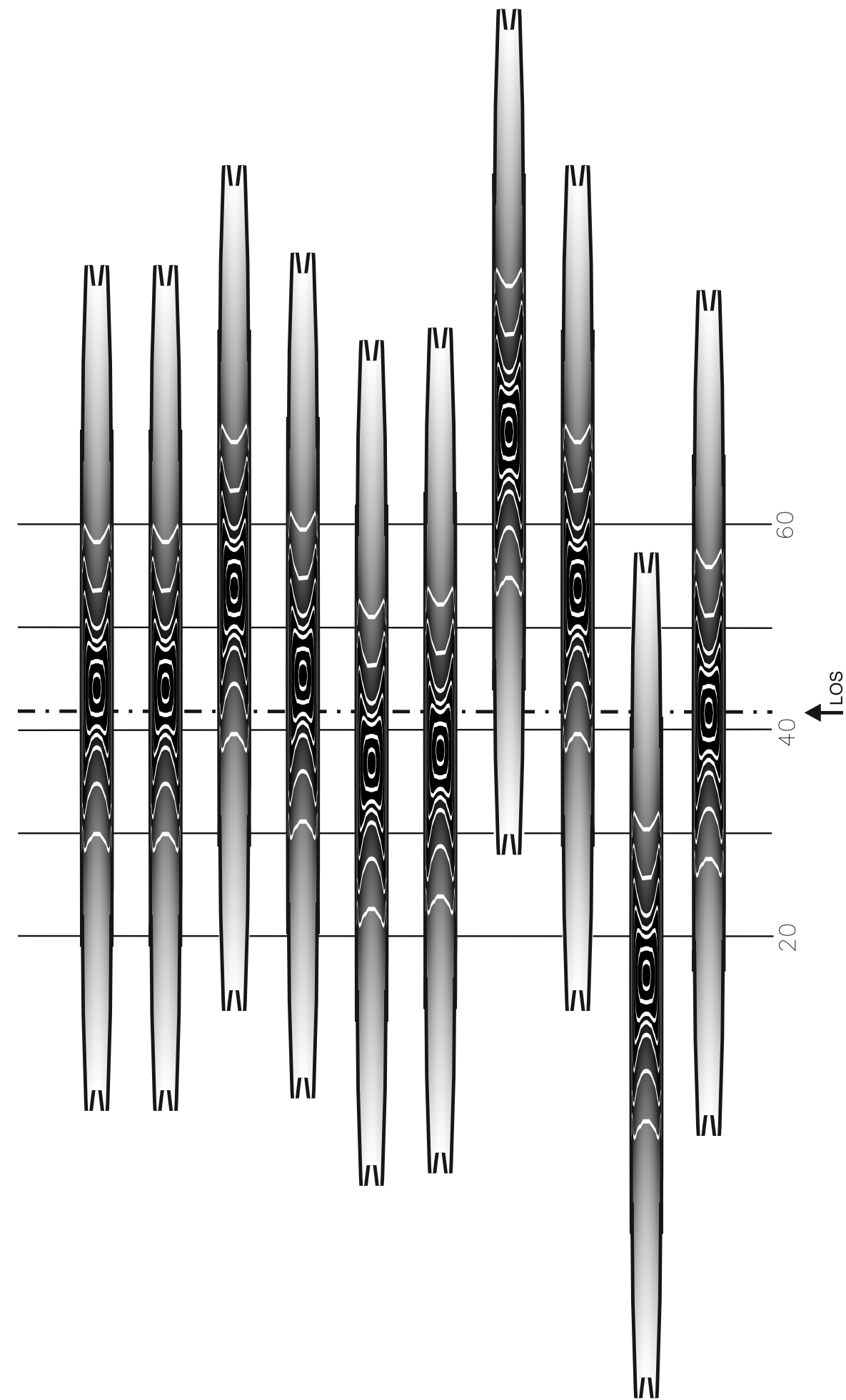

Fig. 2. The realization of the multi-thread model. Arrows indicate the direction and magnitude of LOS velocities. White solid lines represent the iso-contours of the density $(5.0,2.5$, $1.0,0.5,0.25,0.1$, and $0.05 \times 10^{-13} \mathrm{~g} \mathrm{~cm}^{-3}$, starting from the centre of the thread). The grey-scale represent the temperature, where black colour indicates the minimum and white colour the maximum temperature. The dotdashed line represents the centre of the foremost thread (position 42) and the thin solid lines indicate positions 20 to 60 .

\section{Discussion and conclusions}

Our multi-thread model with randomly assigned LOS velocities to individual threads produces substantial asymmetries of the line profiles even though the LOS velocities are only of the order of $10 \mathrm{~km} \mathrm{~s}^{-1}$. We have performed additional tests with the LOS velocities from intervals $\langle-5,5\rangle \mathrm{km} \mathrm{s}^{-1}$ and $\langle-15,15\rangle \mathrm{km} \mathrm{s}^{-1}$ and the overall pattern does not alter significantly. The asymmetries however tend to be more pronounced for higher LOS velocities. Our results also show that the Lyman- $\alpha$ profiles may exhibit an opposite asymmetry to that of the higher Lyman lines.

If we assume that the slit of the SUMER detector is oriented along the length of the foremost thread of the multithread model, Figs. A.1 to A.4 would represent an example of 

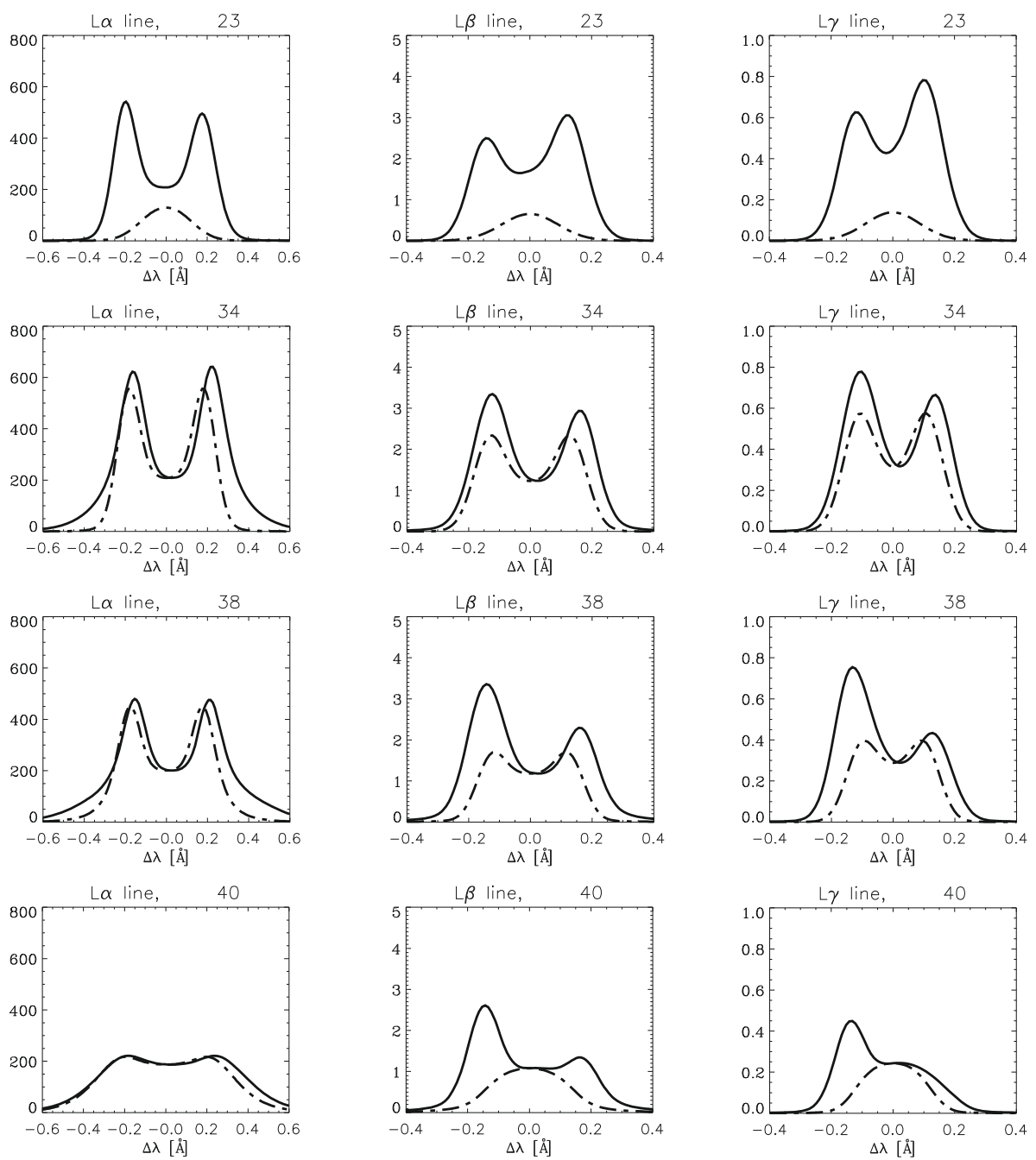

Fig. 3. Profiles of the Lyman- $\alpha$ to Lyman- $\gamma$ lines at the positions $23,34,38$, and 40 . The full lines represent profiles that result from the set of 10 individual threads with random LOS velocities. Dot-dashed lines represent profiles produced by a single thread model at the same positions. The intensity is in $10^{-10} \mathrm{erg} \mathrm{s}^{-1} \mathrm{~cm}^{-2} \mathrm{sr}^{-1} \mathrm{~Hz}^{-1}$. The wavelength scale $\Delta \lambda$ is given with respect to the line centre in the rest frame for each of the lines (Lyman- $\alpha$ centre at $1215.67 \AA$, Lyman $\beta$ centre at $1025.72 \AA$, and Lyman- $\gamma$ centre at $972.54 \AA$ ). the observed spectrum. Thus we could compare the characteristics of this synthetic spectrum with observed one. Figure 5 shows an example of the observed prominence spectra along the SUMER slit. Lyman- $\alpha$ and Lyman- $\beta$ lines are displayed for pixels 19 to 21 along the slit. More profiles can be found in Fig. A.6, where we show Lyman- $\alpha$ and Lyman- $\beta$ lines for pixels 10 to 27 along the slit. These observational data represent a part of a quiescent prominence observed on May 25, 2005 by SUMER (detector A) during the MEDOC coordinated campaign between SOHO and ground-based instruments (MEDOC campaign No. 15, May 16-29, 2005). The centre of the SUMER slit was pointed at solar coordinates $X=972^{\prime \prime}$ and $Y=168^{\prime \prime}$ and the slit was oriented in the north-south direction. Lyman- $\alpha$ observations started at 19:10:03 UT and Lyman- $\beta$ at 19:12:23 UT, both with the exposure time of $115 \mathrm{~s}$. The observational data presented here are part of the data set used in the study of Gunár et al. (2007b). We note that in the latter study we selected only symmetrical profiles.

The data were properly calibrated using standard Solar-Soft procedures, including the procedure delta_pixel.pro for a correction of shifts of pixel positions for different spectral lines. The slit positions indicated in Figs. 5 and A.6 therefore correspond to the same position at the observed quiescent prominence. The observed data exhibit changes of the profile asymmetries along the slit for both spectral lines with a rather random pattern. Moreover, the asymmetries have in several cases an opposite sense at the same position on the slit for different spectral lines (see pixels 19 to 21 or pixel 24). An example of observed data shown in Figs. 5 and A.6 corresponds to the same prominence studied by Vial et al. (2007), who found similar behaviour of the line asymmetries of the Lyman- $\alpha$ and Lyman- $\beta$ lines. In future work, we plan to employ statistical methods to compare the behaviour of the synthetic and observed asymmetrical line profiles.

\subsection{The nature of the LOS velocities}

The aim of the present investigation was to show that rather small LOS velocities of the order of $10 \mathrm{~km} \mathrm{~s}^{-1}$ can produce relatively large asymmetries that are actually observed in the Lyman lines. Concerning the nature of these velocities, we note that even quiescent prominences are very dynamic when one looks at their fine structures. $\mathrm{H} \alpha$ movies obtained by SOT (Solar Optical Telescope) onboard the Hinode satellite, show oscillations and random motions of fine-structure threads. These motions are considered to represent random oscillatory motions of the dipped fine-structure magnetic field. The LOS component of these motions can produce the type of velocities used in our modelling. There are also flows along the magnetic field in quiescent prominences (Schmieder et al. 1991; Zirker et al. 1998; Lin et al. 2003), which have typical velocities of $10 \mathrm{~km} \mathrm{~s}^{-1}$. These flows would not produce any LOS velocities in the specific model that we use here. But if the LOS direction is not exactly perpendicular to the field lines, one can obtain non-zero LOS components 

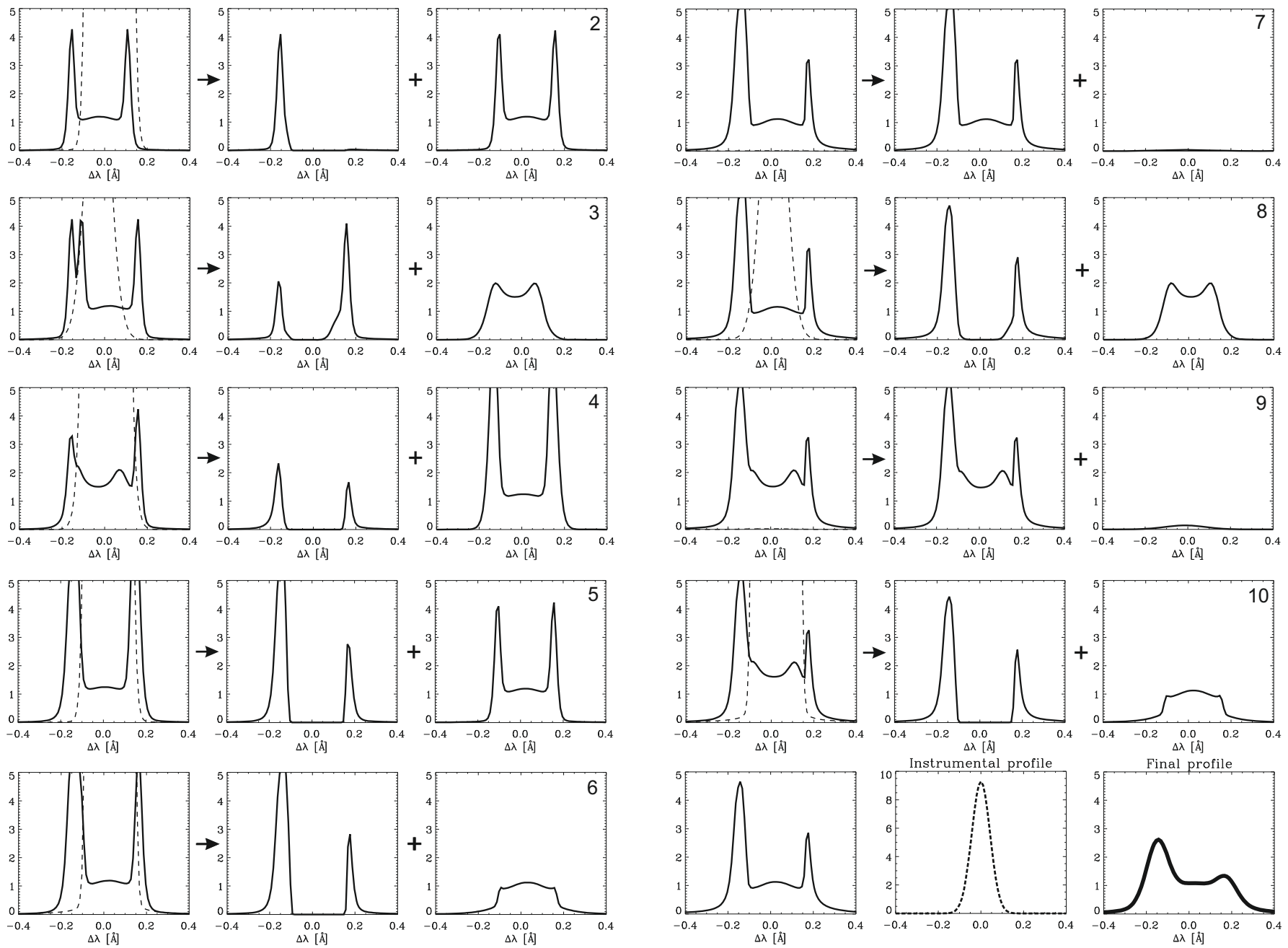
column of each part gives the line profile as seen in front of the thread $n$ (solid line) and also the optical depth profile of the thread $n+1$ (dashed line). The second column shows the line profile after absorption by the thread $n+1$, and the third column gives the contribution of that thread. The sum of these two profiles is then the new line profile. The final row at the right hand side shows the resulting profile without instrumental effects (first plot), the instrumental profile (second plot) and the final line profile after the convolution with the instrumental profile (third plot). The scaling of the intensities is the same in all plots $\left(10^{-10} \mathrm{erg} \mathrm{s}^{-1} \mathrm{~cm}^{-2} \mathrm{sr}^{-1} \mathrm{~Hz}^{-1}\right)$ and the numbers on the axis also indicate the values of the optical depths. The wavelength scale is given with respect to the line centre of Lyman- $\beta$ in the rest frame at $1025.72 \AA$.
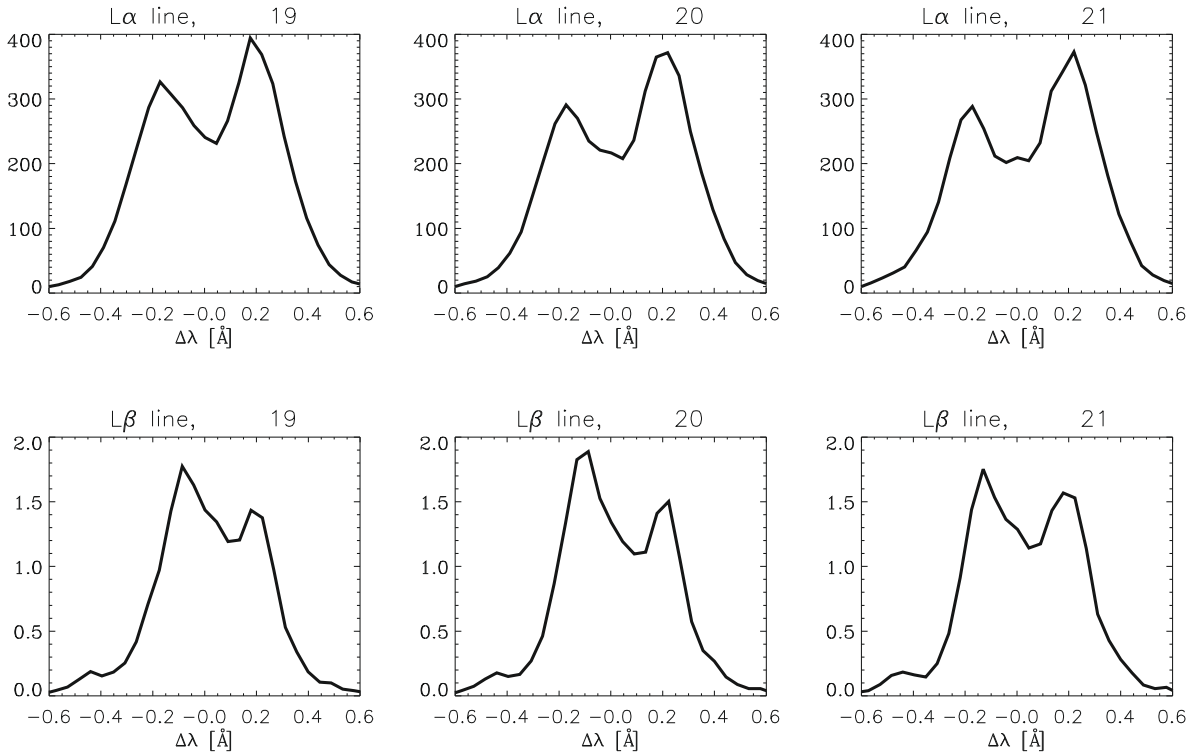

Fig. 5. Lyman- $\alpha$ and Lyman- $\beta$ prominence spectra observed on May 25, 2005 by SUMER. The positions 19 to 21 along the slit are shown. The scaling of the intensities in all plots is given in units of $\left(10^{-10} \mathrm{erg} \mathrm{s}^{-1} \mathrm{~cm}^{-2} \mathrm{sr}^{-1} \mathrm{~Hz}^{-1}\right)$. The wavelength scale is given with respect to the line centre of Lyman- $\alpha$ and Lyman- $\beta$ in the rest frame at $1215.67 \AA$ and $1025.72 \AA$, respectively. 
of the velocity: assuming e.g. an angle of $60^{\circ}$ between LOS and magnetic field, one obtains a LOS velocity that amounts to $50 \%$ of the total velocity. Under these circumstances, we also expect large line asymmetries. This additional aspect has not been taken into account in the present modelling, but we plan to study this in a future paper.

Acknowledgements. The authors thank P. Schwartz for reduction and correction of the observed SUMER data. The authors acknowledge support from the International Space Science Institute, Bern, Switzerland to the International Team 123. S.G. and P.H. acknowledge the support from the MPA Garching; U.A. thanks for support from the Ondřejov Observatory. This work was also supported by ESA-PECS project No. 98030 and the institutional project AV0Z10030501. SOHO is a space mission of international cooperation between ESA and NASA.

\section{References}

Anzer, U., \& Heinzel, P. 1999, A\&A, 349, 974

Chae, J., Schühle, U., \& Lemaire, P. 1998, ApJ, 505, 957

Fontenla, J. M., Rovira, M., Vial, J.-C., \& Gouttebroze, P. 1996, ApJ, 466, 496 Gunár, S., Teriaca, L., Heinzel, P., \& Schühle, U. 2006, in Proc. SOHO 17 Conf., ESA SP-617

Gunár, S., Heinzel, P., \& Anzer, U. 2007a, A\&A, 472, 929

Gunár, S., Heinzel, P., Schmieder, B., Schwartz, P., \& Anzer, U. 2007b, A\&A, 472,929

Gouttebroze, P., Heinzel, P., \& Vial, J.-C. 1993, A\&A, 99, 513

Heasley, J. N., \& Mihalas, D. 1976, ApJ, 205, 273
Heinzel, P. 2007, in The Physics of Chromospheric Plasmas, ed. P. Heinzel, I. Dorotovič, \& R. J. Rutten, ASP-368, 271

Heinzel, P., \& Anzer, U. 2001, A\&A, 375, 1082

Heinzel, P., \& Anzer, U. 2005, in Solar Magnetic Phenomena, ed. A. Hanslmeier, A. Veronig, \& M. Messerotti (Dordrecht: Springer) 115, Astron. Astrophys. Space Sci. Lib., 320

Heinzel, P., Schmieder, B., Vial, J. C., \& Kotrč, P. 2001, A\&A, 370, 281

Heinzel, P., Anzer, U., \& Gunár, S. 2005, A\&A, 442, 331

Heinzel, P., Schmieder, B., \& Vial, J. C. 2006, in Proc. SOHO 17 Conf., ESA SP-617

Kippenhahn, R., \& Schlüter, A. 1957, Z. Astrophys., 43, 36

Kucera, T. A., Aulanier, G., Schmieder, B., Mein, N., \& Vial, J.-C. 1999, Sol. Phys., 186, 259

Labrosse, N., \& Gouttebroze, P. 2004, ApJ, 617, 614

Lin, Y., Engvold, O., \& Wiik, J. E. 2003, Sol. Phys., 216, 109

Madjarska, M. S., Vial, J.-C., Bocchialini, K., \& Dermendjiev, V. N. 1999, in Proceedings of the 8th SOHO Workshop, ed. B. Kaldeich, \& J.-C. Vial, ESA SP-446, 467

Patsourakos, S., \& Vial, J.-C. 2002, Sol. Phys., 208, 253

Schmieder, B., Raadu, M. A., \& Wiik, J. E. 1991, A\&A, 252, 353

Schmieder, B., Heinzel, P., Vial, J.-C., \& Rudawy, P. 1999, Sol. Phys., 189, 109

Schmieder, B., Gunár, S., Heinzel, P., \& Anzer, U. 2007, Sol. Phys., 241, 53

Tandberg-Hanssen, E. 1995, The Nature of Solar Prominences (Dordrecht: Kluwer Acad. Publ.)

Vial, J.-C. 1982, ApJ, 254, 780

Vial, J.-C., Ebadi, H., \& Ajabshirizadeh, A. 2007, Sol. Phys., 246, 327

Wiik, J. E., Dammasch, I. E., Schmieder, B., \& Wilhelm, K. 1999, Sol. Phys., 187,405

Wilhelm, K., Lemaire, P., Curdt, W., et al. 1995, Sol. Phys., 170, 75

Zirker, J. B., Engvold, O., \& Martin, S. F. 1998, Nature, 396, 440 
Appendix A: Online material
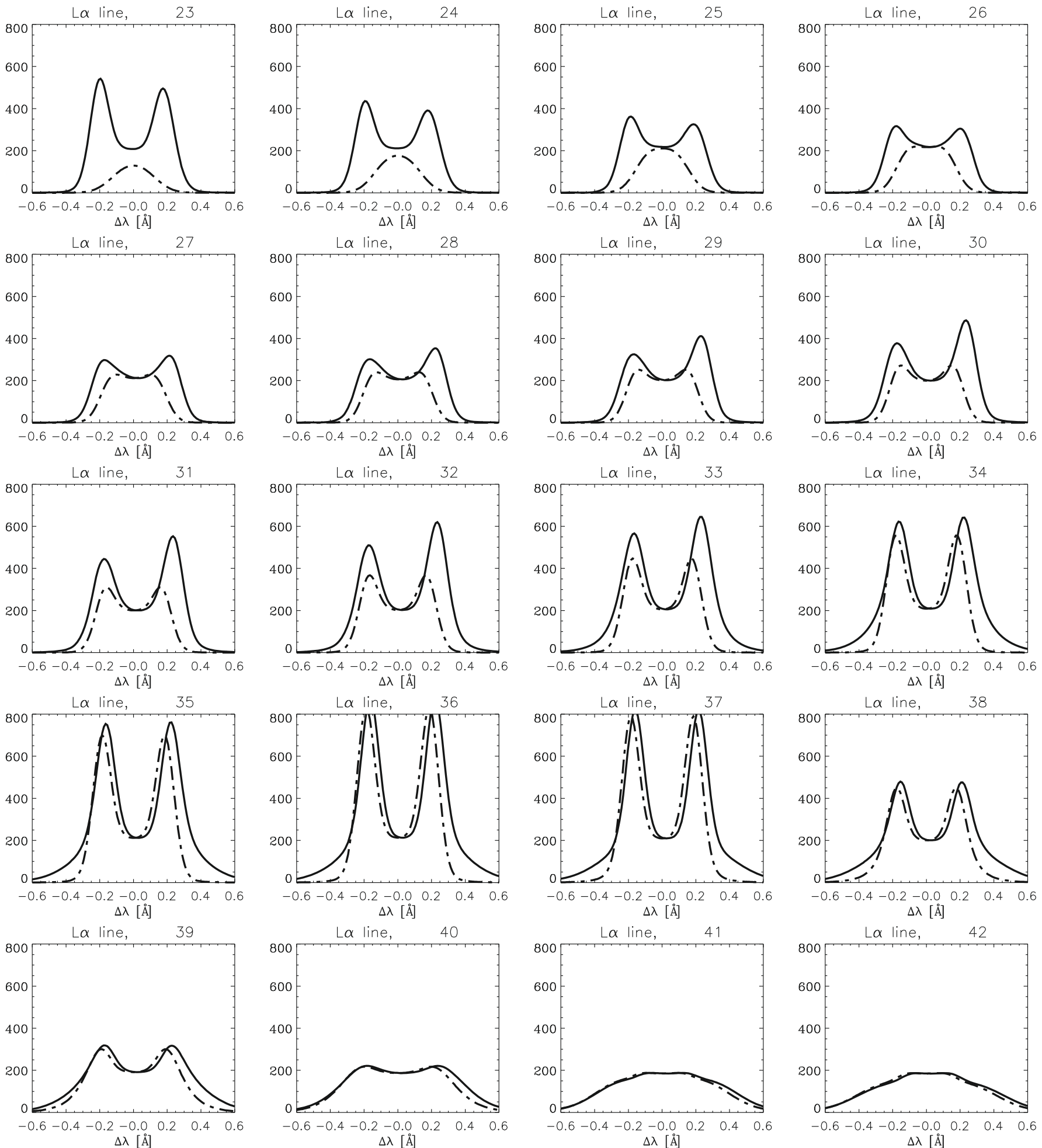

Fig. A.1. Lyman- $\alpha$ profiles at positions 23 to 42 along the length of the thread, position 42 represents the centre of the thread. Full lines represent the resulting profiles from the set of 10 threads. Dot-dashed lines represent the profiles emerging from a single $2 \mathrm{D}$ thread model. The intensity is in $10^{-10} \mathrm{erg} \mathrm{s}^{-1} \mathrm{~cm}^{-2} \mathrm{sr}^{-1} \mathrm{~Hz}^{-1}$. The wavelength scale $\Delta \lambda$ is given with respect to the line centre of Lyman- $\alpha$ in the rest frame at $1215.67 \AA$. 
S. Gunár et al.: Lyman-line asymmetries, Online Material p 2
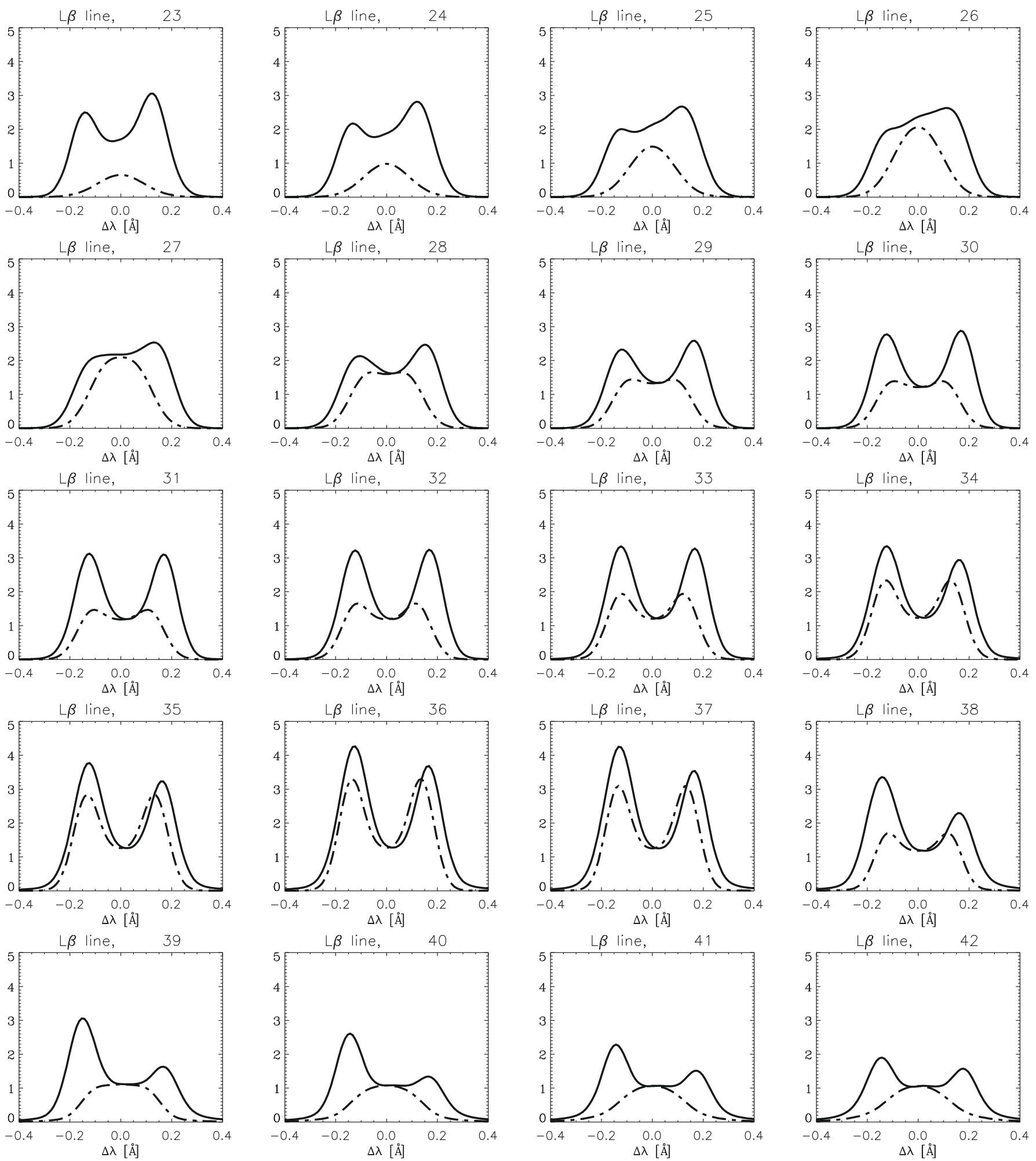

Fig. A.2. Same as in Fig. A.1 but for Lyman- $\beta$ line (line centre at $1025.72 \AA$ ). 
S. Gunár et al.: Lyman-line asymmetries, Online Material p 3
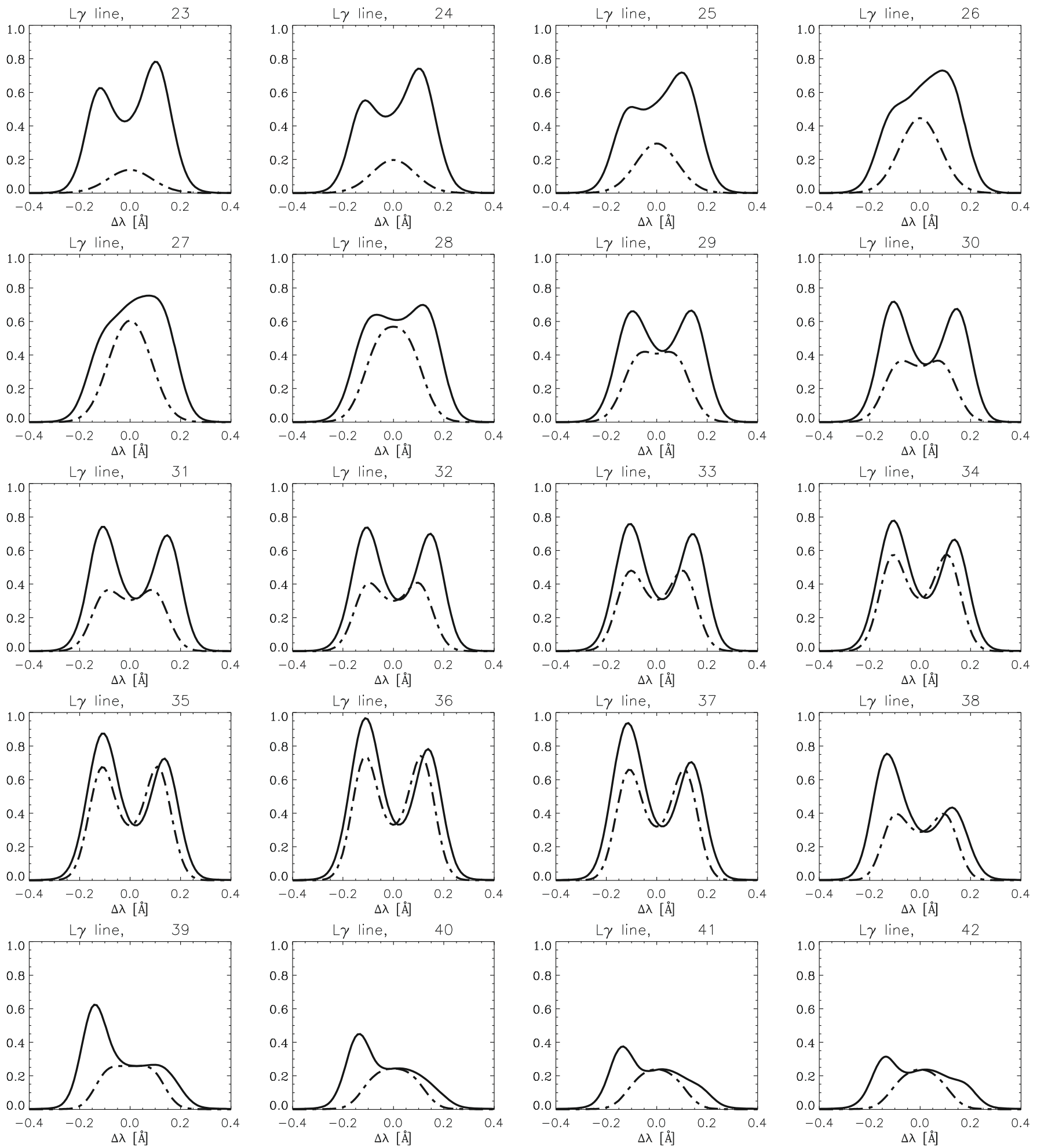

Fig. A.3. Same as in Fig. A.1 but for Lyman- $\gamma$ line (line centre at $972.54 \AA$ ). 
S. Gunár et al.: Lyman-line asymmetries, Online Material p 4
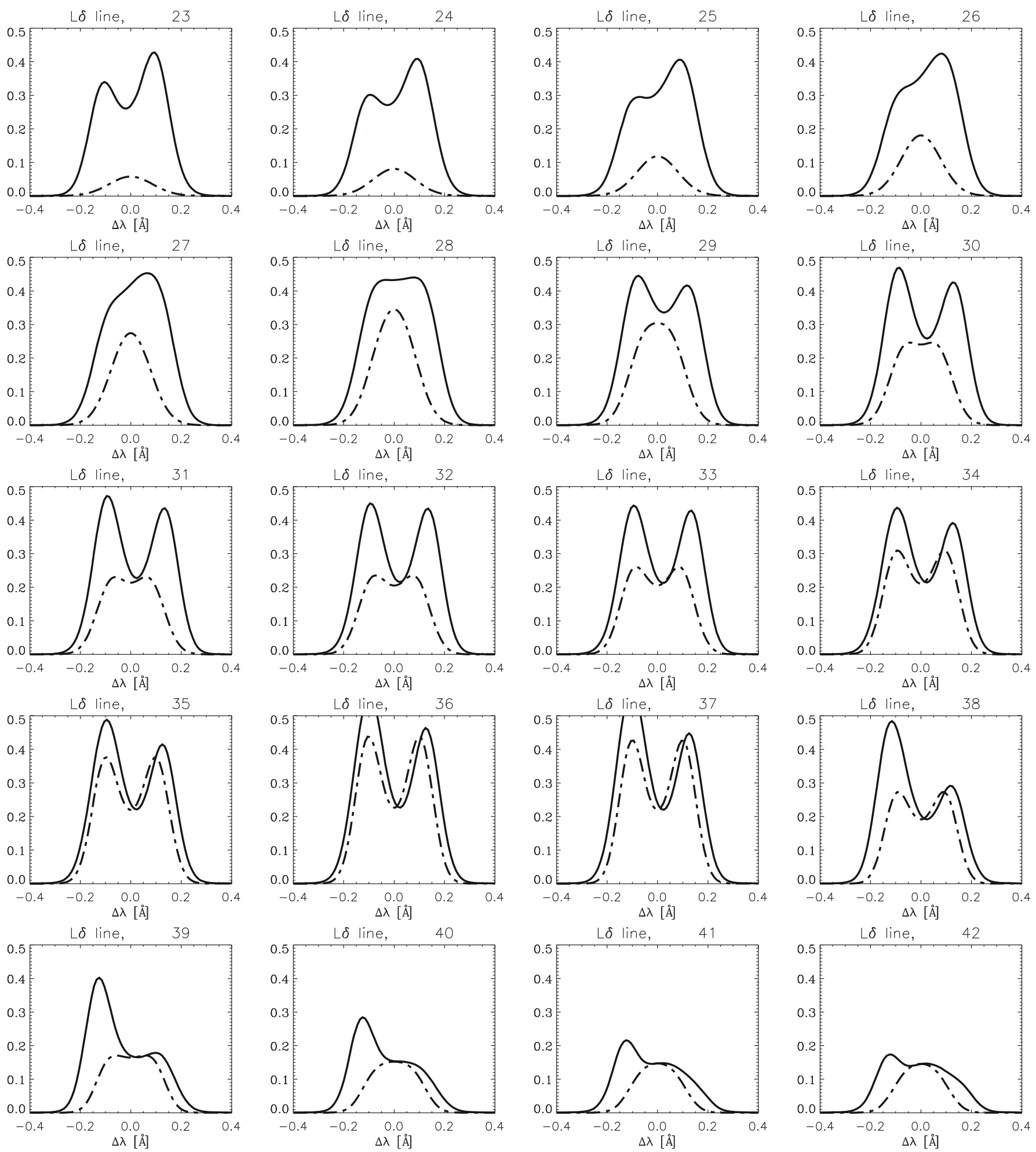

Fig. A.4. Same as in Fig. A.1 but for Lyman- $\delta$ line (line centre at $949.74 \AA$ ). 
S. Gunár et al.: Lyman-line asymmetries, Online Material p 5
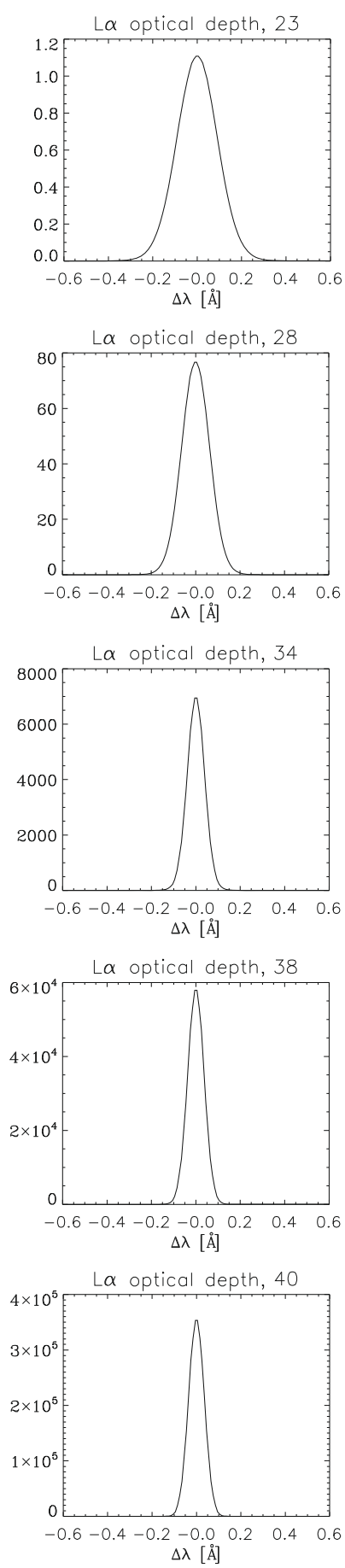
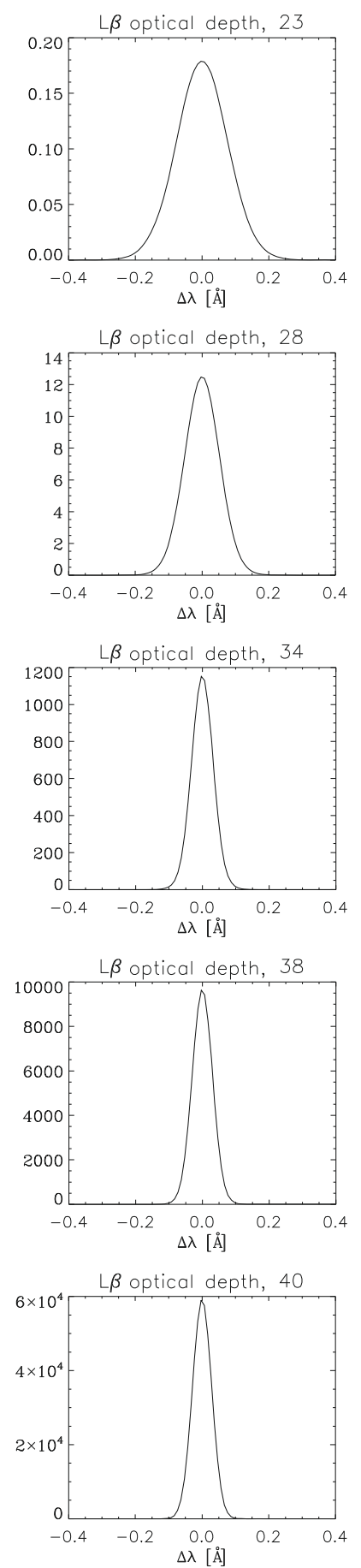
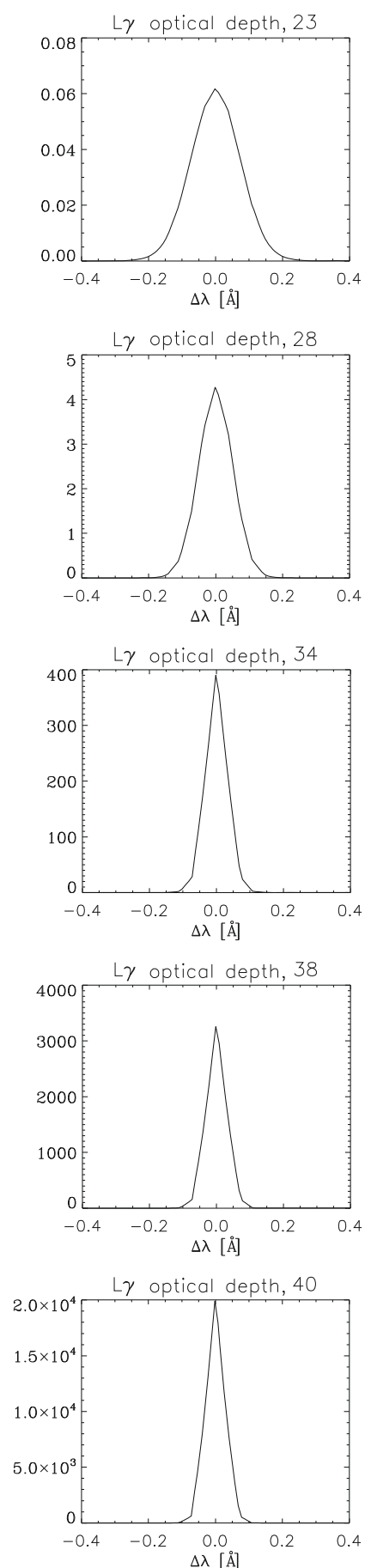
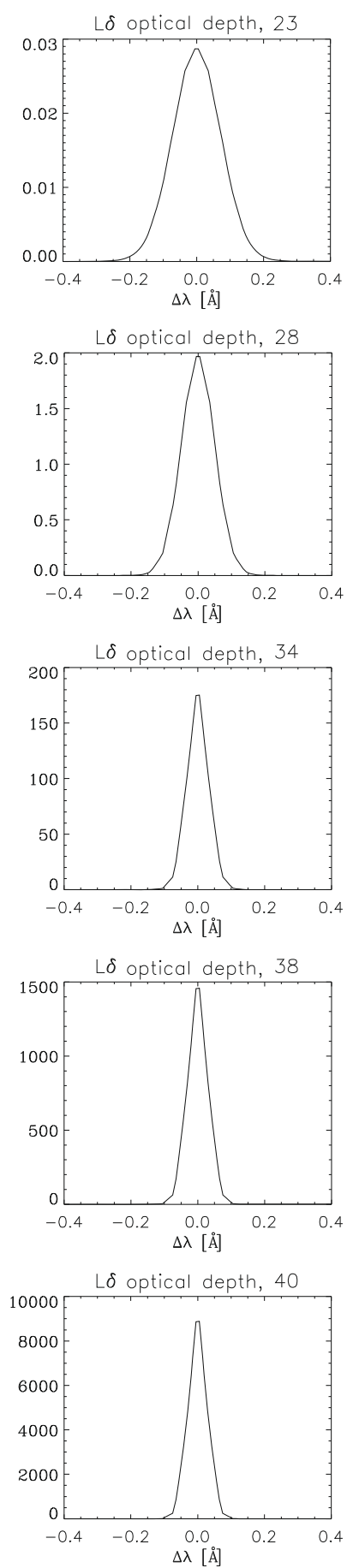

Fig. A.5. Optical depth profiles of Lyman- $\alpha$ to Lyman- $\delta$ lines at position 23, 28, 34, 38, and 40 along the length of the thread. Each column represents the optical depth profiles of one spectral line. The wavelength scale $\Delta \lambda$ is given with respect to the centre of a given line in the rest frame (Lyman- $\alpha$ centre at 1215.67 $\AA$, Lyman- $\beta$ centre at 1025.72 $\AA$, Lyman- $\gamma$ centre at $972.54 \AA$, and Lyman- $\delta$ centre at $949.74 \AA$ ). 
S. Gunár et al.: Lyman-line asymmetries, Online Material p 6
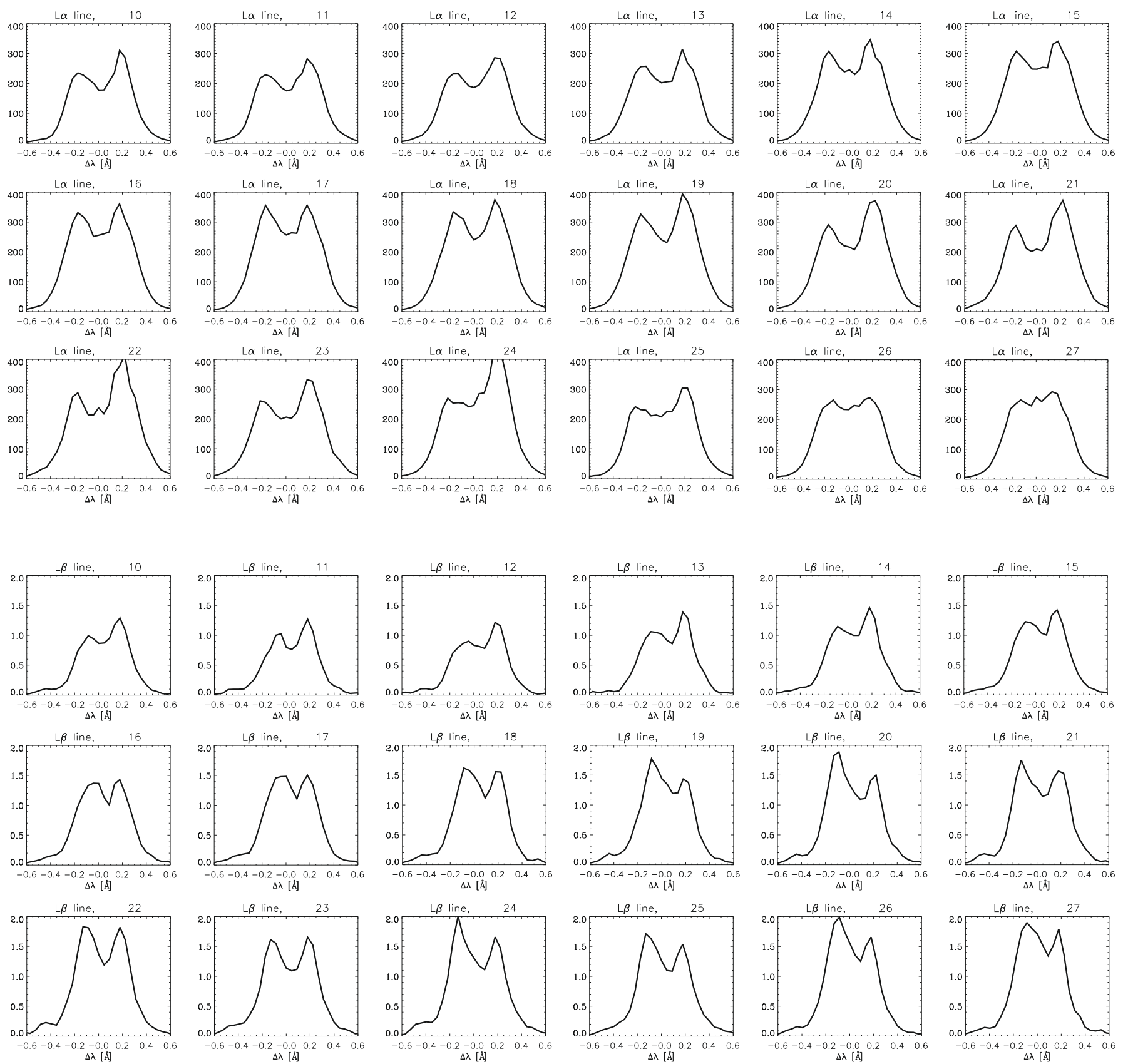

Fig. A.6. Lyman- $\alpha$ and Lyman- $\beta$ prominence spectra observed on May 25, 2005 by SUMER. The positions 10 to 27 along the slit are shown. The scaling of the intensities in all plots is given in units of $\left(10^{-10} \mathrm{erg} \mathrm{s}^{-1} \mathrm{~cm}^{-2} \mathrm{sr}^{-1} \mathrm{~Hz}^{-1}\right)$. The wavelength scale is given with respect to the line centre of Lyman- $\alpha$ and Lyman- $\beta$ in the rest frame at $1215.67 \AA$ and $1025.72 \AA$, respectively. 